\title{
Evidence of Dispersion in a Water-Saturated Granular Sediment
}

\author{
P. S. Wilson \\ Mechanical Engineering Department \\ The University of Texas at Austin \\ Austin, TX 78712-0292, USA \\ Email: pswilson@mail.utexas.edu
}

\author{
J. C. Wilbur, R.A. Roy and W. M. Carey \\ Department of Aerospace and Mechanical Engineering \\ Boston Univesity \\ Boston, MA 02215, USA
}

\begin{abstract}
An experiment was conducted to measure the sound speed in a water-saturated granular sediment within a range of frequencies where dispersion is predicted by a number of existing models. Between $2-4 \mathrm{kHz}$, the sound speed was inferred from measurements of the resonance frequencies of a right-cirular-cylindrical container filled with the material. From $20-300 \mathrm{kHz}$, the sound speed was obtained directly from time-of-flight measurements within the same sediment. Dispersion consistent with the Williams Effective Density Fluid Model was observed.
\end{abstract}

\section{INTRODUCTION}

A complete understanding of sound propagation in water-saturated granular sediments has not been achieved. A number of experimental studies have appeared in the literature that report either direct or indirect evidence of sound speed dispersion in this medium [1-6]. Such results generally support the Biot-Stoll model (and its variants) [7-12] for sound propagation. The Biot-derived models predict a low frequency sound speed, followed by an increase to a higher sound speed beyond some transition frequency. There are also a number of experimental studies [13, 14] that found no evidence of sound speed dispersion and support Hamilton's dispersionless fluid model [15]. The dispersion predicted by the Biot models occurs over a range of about two orders of magnitude in frequency (from around $100 \mathrm{~Hz}$ to $10 \mathrm{kHz}$ ) and therefore, it is experimentally difficult to obtain measurements on a uniform body of sediment using a single technique that spans the entire range of dispersion. Further, the predicted dispersion is on the order of $10 \%$ and achieving experimental accuracy significantly greater than this has been difficult. Surface and volume inhomogeneities in natural sediments also cause uncertainty in experimental measurements.

A series of laboratory experiments designed to overcome some of these difficulties were performed on a water-saturated sand sediment. The sediment was contained within a thin-walled cylindrical vessel made that was large enough to permit time-of-flight sound speed measurements within the bulk sediment above $20 \mathrm{kHz}$. Lower frequency sound speeds were inferred from the measured resonance frequencies of the cylindrical sediment-filled container. For this analysis, transverse waves within the sediment were ignored. The sediment was modeled as a fluid, but the sediment sound speed was not constrained to be constant. To leading order, the walls of the cylinder approached a pressure release boundary. The cylinder was placed upon a layer of insulation foam, which formed a pressure release lower boundary condition. The water/air interface provided a pressure release boundary at the top of the sediment. Therefore, a pressure release waveguide model was used to relate the measured resonance frequencies to the effective sediment sound speed. In order to identify individual modes, and hence infer frequency-dependent sound speeds from resonances, both symmetric and anti-symmetric excitation of the sediment cylinder was used. The phase of the received acoustic pressure, relative to the drive signal was also employed to aid in mode identification. Dispersion was observed and these results are presented and compared to existing theory. The effects of hoop stress imparted by the cylindrical walls of the sediment container is also discussed.

\section{DESCRIPTION OF THE EXPERIMENT}

\section{A. Sediment Container}

The sediment was contained in a right-circular-cylindrical tank that had walls made of high-density polyethylene. The mean inner diameter was $0.67 \mathrm{~m}$, the height of the sediment within the vessel was $0.80 \mathrm{~m}$, and the mean wall thickness was $0.48 \mathrm{~cm}$. The tank, which had a flat bottom, was placed upon a $10 \mathrm{~cm}$ thick styrofoam panel.

The sediment was prepared in the following manner. Filtered fresh water was added to the tank. The water was degassed in the tank by heating it to $60^{\circ} \mathrm{C}$ using several imersion heaters. Circulation was imposed during this process with a motorized stirring impeller. The water was kept at $60^{\circ} \mathrm{C}$ for several hours. Then, approximately $500 \mathrm{~kg}$ of a commercial silica blasting sand was slowly added to the water, as the stirring continued. The sediment was then let to cool naturally to room temperature, which took more than 24 hours. The supplier of the sand reported a grain size range of $150-600 \mu \mathrm{m}$. We conducted a sieve analysis on approximately $500 \mathrm{ml}$ of the sand that confirmed the suplier-reported range and yeilded a mean grain size of $300 \mu \mathrm{m}$.

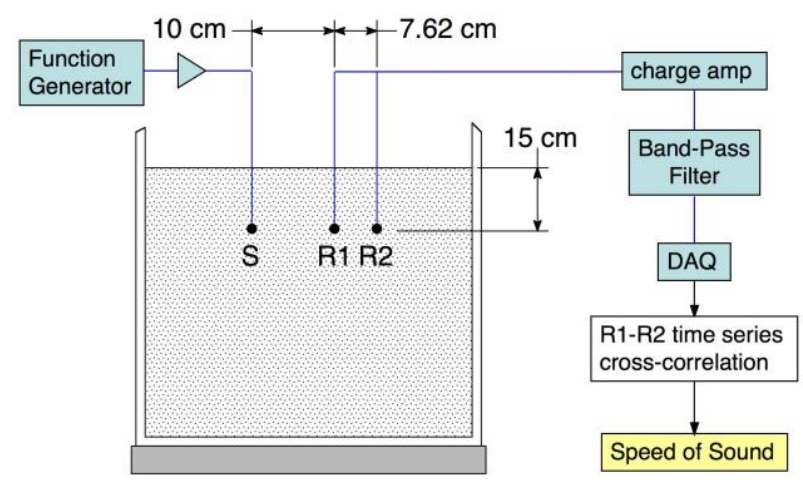

Fig. 1. A schematic diagram of the time-of-flight measurement apparatus.

\section{B. High-Frequency Time-of-Flight Measurements}

The sound speed within the sediment was measured using a time-of-flight technique at 20,50, 100 and $300 \mathrm{kHz}$. A schematic diagram of this experiment is shown in Fig. 1. The signal source was a function generator programmed to produce 
sinusoidal pulses of various length, depending upon the frequency, to avoid multipath interference. The source signal was band-pass filtered ( $24 \mathrm{~dB} /$ octave) at the pulse center frequency with a Krohn-Hite model 34A filter and then amplified by a Krohn-Hite model 7500 wide-band power amplifier and directed to a Brüel \& Kjær model 8103 miniature hyrdophone, operating as a source (labeled $\mathrm{S}$ in

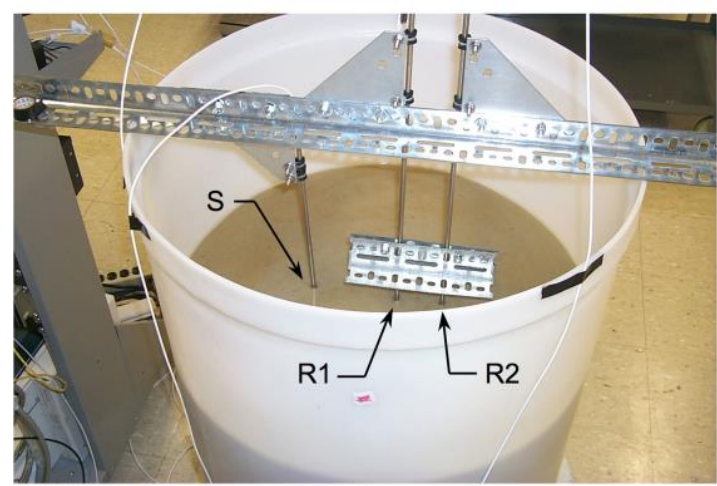

Fig. 2. The sediment container, the hydrophone mounting framework, and the source $(\mathrm{S})$ and receiver $(\mathrm{R} 1, \mathrm{R} 2)$ tansducers deployed in the sediment are shown.

Fig. 1). Two more 8103 hydrophones were positioned $10 \mathrm{~cm}$ and $17.6 \mathrm{~cm}$ away from the source on a horizontal line (parallel to the sediment surface), at a depth of $15 \mathrm{~cm}$. The latter two hydrophones acted as receivers (labeled R1 and R2 in Fig. 1). The relative positions of the source and receivers were ensured to within $+/-0.25 \mathrm{~mm}$ by mounting each hydrophone and its cable inside a stainless steel tube, as shown in Fig. 2. These tubes were then mounted to a steel frame and fastened securely in place. This way, the relative positions could be set and meausred with a micrometer caliper prior to inserting the hydrophones into the sediment. The rigidity of the mounting scheme was sufficient to prevent deflection during the inserting process. The hydrophone-enclosing tubes were filled with degassed water, to minimize acoustic disturbance of the sediment.

The signals from R1 and R2 were conditioned with a Brüel \& Kjær model 2692 charge amplifier, then band-pass filtered (24 $\mathrm{dB} /$ octave) at the pulse center frequency with Krohn-Hite model 34A filters. The filtered source and receive signals were acquired with a digital oscilloscope. At each frequency, 100 pings were collected and the average was sent to a PC for processing. The R1 and R2 signals were cross-correlated to obtain the time-of-flight and then sound speed was calculated from the known sensor separation distance. The uncertainty in sound speed was calculated from the previously stated position uncertainty, and the digitiztion sample rate and found to be between $+/-6.6 \mathrm{~m} / \mathrm{s}$ and $+/-9.9 \mathrm{~m} / \mathrm{s}$ at $300 \mathrm{kHz}$ and $20 \mathrm{kHz}$ respectively.

\section{Low-Frequency Resonance Measurements}

The apparatus for this portion of the experiment is shown in Fig. 3. A custom-fabricated Tonpiltz-type transducer was used as a source. It is described in [16] and has a circular-shaped radiating face with an accelerometer imbedded it, and therefore can be driven at constant velocity amplitude by suitable monitoring and modifcation of the excitation voltage amplitude. The source was placed face-down on the surface of the sediment in one of two positions, to be described later. The source signal was band-limited $(2-5 \mathrm{kHz})$ pseudo-random noise, provided by a Hewlett-Packard 89410A vector signal analyzer (VSA) and amplified by a Crown CE 1000 power amplifier. The source amplitude was modified onboard the VSA to produce a velocity amplitude that was uniform across the frequency range, as disucssed above. The acoustic pressure was measured in the sediment with B\&K 8103 hydrophones at two positions, labeled R1 and R2 in Fig. 3. The depth was $6.7 \mathrm{~cm}$ and each sensor was positioned $10 \mathrm{~cm}$ from the vessel wall. When observed from above, R1-S-R2 formed a straight line. The signals from R1 and

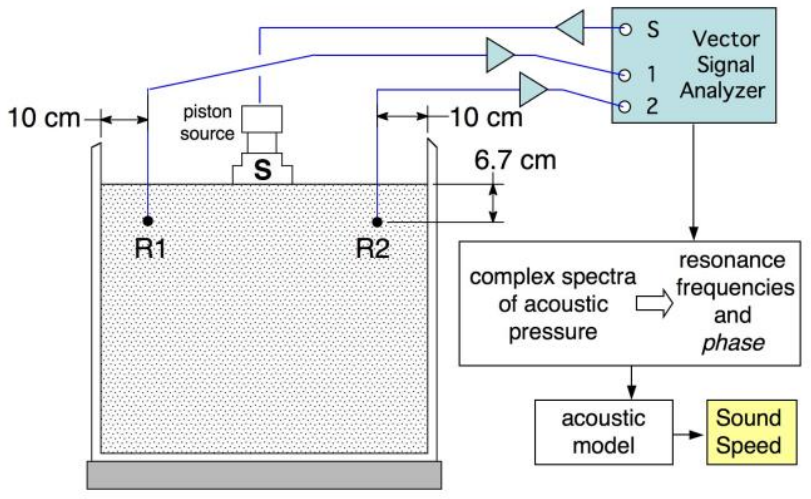

Fig. 3. A schematic diagram of the resonance frequency apparatus.

R2 were conditioned by the B\&K 2692 charge amp, which included band-pass filtering between $10-10 \mathrm{k} \mathrm{Hz}$, and digitized by the VSA. The complex spectrum of each hydrophone signal (with phase referenced to the excitation signal) was calculated onboard the analyzer using 100 spectral averages and converted to complex acoustic pressure spectra using the calibrated hydrophone sensitivities.

\section{ACOUSTIC MODEL OF SEDIMENT AND CONTAINER}

An acoustic model of the sediment/container system is needed to infer sound speeds from measured resonance frequencies. In this analysis, the sediment was considered to be an effective fluid with wave number $k$, which is a valid approach for granular sediments [12]. We assume the phase speed can be frequency-dependent, but $\operatorname{Im}[k]<<\operatorname{Re}[k]$, so that the resonance frequencies dependent primarily upon the phase speed and have weak dependence upon the attenuation. Transverse waves in the sediment were ignored.

Initially we ignore the container walls and conider the sides of the cylinder to have a pressure-release boundary condition under the following justification. 1) Typical values of the specific acoustic impedance of the materials involved are given in Table 1. Based on these values and the wall thickness $(0.48 \mathrm{~cm})$, the three-medium reflection coefficient $R$, given by Eq. 6.3.7 in [17], for pressure waves incident upon the wall, is $R=1.00 \angle 176.5^{\circ} \pm 1.5^{\circ}$ between $2-5 \mathrm{kHz}$. 2) This was qualitat-ively verified by the observation of inverted reflections from the walls during the time-of-flight measurements, and 3) by the absence of plane-wave longitudinal mode resonances in the system. The air-sediment interface at the top of the 
container is clearly pressure-release. Finally, the container was placed upon a $10 \mathrm{~cm}$-thick styrofoam building insulation panel to approximate a pressure release boundary condition on the bottom. The acoustic pressure in a right-circular cylinder with $p=0$ boundary conditions at all surfaces is

$$
p_{m n N}(r, \theta, z, t)=A_{m n N} J_{m}\left(\alpha_{m n} r / a\right) \cos \left(m \theta-\theta_{0 m}\right) \sin (N \pi z / h),
$$

and has resonance frequencies

$$
f_{m n N}=\frac{c}{2} \sqrt{\left(\frac{\alpha_{m n}}{\pi a}\right)^{2}+\left(\frac{N}{h}\right)^{2}}
$$

TABLE 1

SPECIFIC ACOUSTIC IMPEDANCE $(\mathrm{Pa} \cdot \mathrm{s} / \mathrm{m})$

\begin{tabular}{ll}
\hline Sediment & $3.5 \times 10^{6}$ \\
tank wall material, HDPE & $2.5 \times 10^{6}$ \\
air & 415 \\
\hline
\end{tabular}

where $\alpha_{m n}$ is the $n$-th root of the Bessel function $J_{m}(x), c$ is the speed of sound in the sediment, $a$ is the tank radius, $h$ is the sediment height. A plot of resonance frequencies vs. longitudinal mode number $N$ is shown in Fig. 4. for the lowest 6 mode families. Five modes, in order of ascending frequency, are labeled with roman numerals from I to V. Note that above mode III, mulitple modes appear with similar eigenfrequencies.

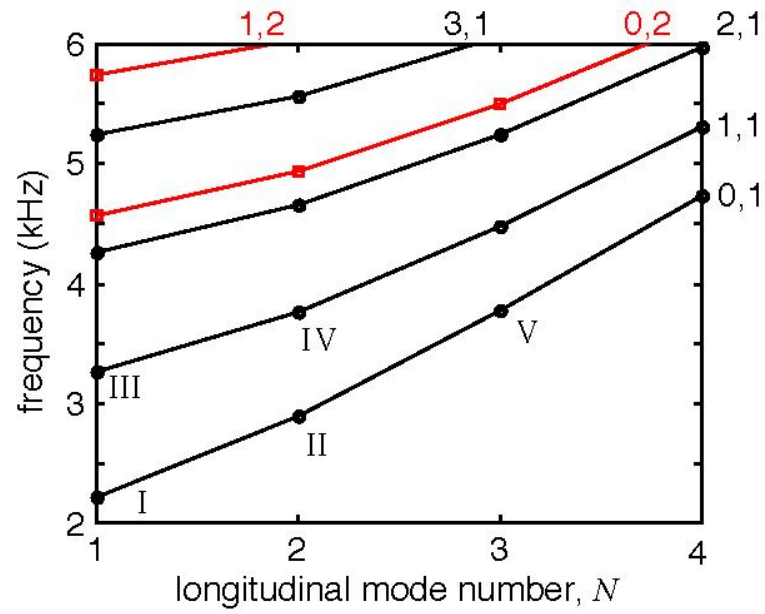

Fig. 4. The resonance frequencies for the 6 lowest-order mode families are shown. The families are labeled $m, n$ around the top and right side of the plot.

Calculation of sound speed from a measured eigenfrequency requires one to identify the specific eigenmode and its $m, n, N$ values. Mode family $0,1, N$ is axisymmetric and mode family $1,1, N$ is non-symmetric about the center of the sediment cylinder, as shown in Fig. 5. These two modes were selectively excited by

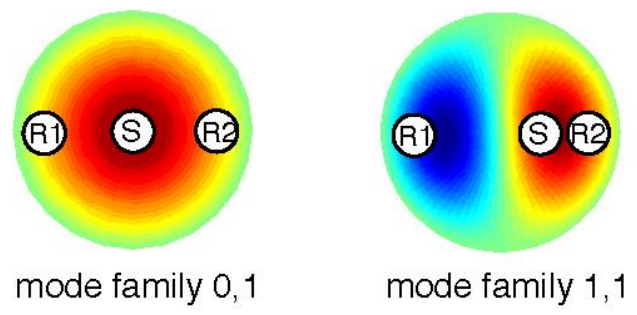

Fig. 5. The mode shapes given by (1) for two mode families are shown. The source positions for each case are labeled $\mathrm{S}$. The receiver positions, which remaim the same in both cases, are labled R1 and R2.

using the source positions shown in Fig. 5. To excite mode family $0,1, N$, the source was placed at the center of the sediment cylinder. To excite mode family $1,1, N$, the source was placed halfway between the center and the container wall. The relative phase of the acoustic pressure observed at R1 and R2 was used to help identify the mode associated with an observed resonance peak. For example, acoustic pressures at R1 and R2 are in phase for modes I,II, and $\mathrm{V}$, and $180^{\circ}$ out of phase for modes III and IV.

\section{RESULTS}

A total of four spectra were obtained during the resonance frequency experiment. These consisted of the acoustic pressure measured at R1 and R2, for both of the source positions shown in Fig. 5. The pressure spectra obtained at R1 are shown in Fig. 6. For the spectral peaks labeled I, II and V, there was less than one degree phase difference between R1 and R2. These three spectral peaks are associated with with mode family $0,1, N$ and correspond with the symmetric eigenmodes labeled I, II and V in Fig. 4. Returning to Fig. 6, the spectral peaks labeled III and IV exhibited an R1-R2 phase difference of $170^{\circ}$. These peaks correspond to the antisymmetric eignemodes modes III and IVshown in Fig. 4 and associated with mode family 1,1,N. Phase speeds were calculated from each of the identified peaks in Fig. 6. Although not shown, similar results were obtained from the sensor at R2.

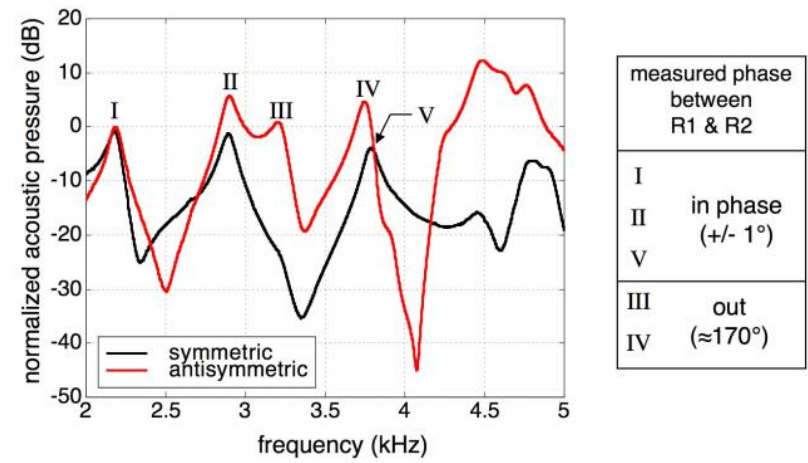

Fig. 6. Acoustic pressure spectra obtained at postion R1 for both symmetric and antisymmetric excitation.

Both the resonance-based sound speeds and the time-of-flight based sounds speeds are shown in Fig. 7. The speeds are normalized by the speed of sound in fresh water at the experimental temperature $\left(23.5^{\circ} \mathrm{C}+/-0.5^{\circ} \mathrm{C}\right)$. The sound speed predicted by the Effective Density Fluid Model [12] is also shown, along with the sediment parameters used in the model. These were primarily taken from [12], but the water sound speed was caluclated from the experimental temperature, and the 
porosity was calculated from a measurement of the saturated-sediment denisty.

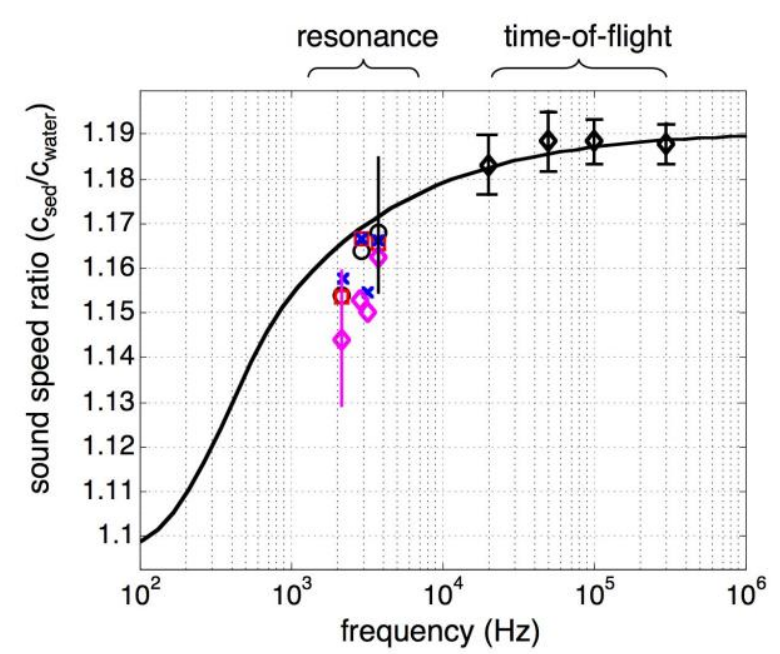

Fig. 7. Sound speed measurements are compared to the EDFM. Error bars represent length and time uncertanties.

TABLE 2. Sediment parameters used as input to the Effective Density Fluid Model of sound propagation.

\begin{tabular}{|l|}
\hline Sediment Parameters \\
(SI units) \\
\hline porosity $=0.37$ \\
sand density $=2650$ \\
water density $=998$ \\
bulk mod. sand $=3.6 \mathrm{e} 10$ \\
$\mathrm{H} 20$ sound speed $=1492$ \\
viscosity $=0.001$ \\
permeability $=1 \mathrm{e}-10$ \\
tortuosity $=1.25$
\end{tabular}

\section{CONCLUSIONS}

Laboratory sound speed measurements were obtained in a water-saturated granular sediment compoosed of commercial silica blasting sand and fresh water. Above $20 \mathrm{kHz}$, a direct time-of-flight method was used and between $2-5 \mathrm{kHz}$, an indirect method was used to infer frequency dependent sound speeds from measured resonance frequencies. Approximately 4\% dispersion was observed across the entire frequency range, which is consisten with the EDFM predictions.

\section{Acknowledgments}

We wish to thank BU and ONR and our families and everyone else.

\section{REFERENCES}

A. Turgut and T. Yamamoto, "Measurement of acoustic wave velocites and attenuation in marine sediments," $J$. Acoust. Soc. Am. 87, pp. 2376-2383 (1990).

[2] A. Maguer, E. Bovio, W.L.J. Fox, and H. Schmidt, "In situ estimation of sediment sound speed and critical angle," $J$. Acoust. Soc. Am. 108, pp. 987-996 (2000).

[3] I. Rosenfeld, W.M. Carey, P.G. Cable, and W.L. Siegmann, "Modeling and analysis of sound transmission in the Strait of Korea," IEEE J. Ocean. Eng. 26, pp. 809-820 (2001).

[4] K.L. Williams, D.R. Jackson, E.I. Thorsos, D. Tang, and S.G. Schock, "Comparison of sound speed and attenuation measured in a sandy sediment to predictions based on the Biot theory of porous media," IEEE J. Ocean. Eng. 27, pp. 413-428 (2002).

[5] R.D. Stoll, "Velocity dispersion in water-saturated granular sediment," J. Acoust. Soc. Am. 111, pp. 785-793 (2002).

[6] N.P. Chotiros, A.P. Lyons, J. Osler, and N.G. Pace, "Normal incidence reflection loss from a sandy sediment," J. Acoust. Soc. Am. 112, pp. 1831-1841 (2002).

[7] M.A. Biot, "Theory of propagation of elastic waves in a fluid-saturated porous solid. I. Low-frequency range," $J$. Acoust. Soc. Am. 28, pp. 168-178 (1956).

[8] M.A. Biot, "Theory of propagation of elastic waves in a fluid-saturated porous solid. II. Higher frequency range," J. Acoust. Soc. Am. 28, pp. 179-191 (1956).

[9] R.D. Stoll and T.K. Kan, "Reflection of acoustic waves at a water-sediment interface," J. Acoust. Soc. Am. 70, pp. 149-156 (1981).

[10] N.P. Chotiros, "Biot model of sound propagation in water-saturated sand," J. Acoust. Soc. Am. 97, pp. 199-214 (1995).

[11] M.J. Buckingham, "Wave propagation, stress relaxation and grain-to-grain shearing in saturated, unconsolidated marine sediments," J. Acoust. Soc. Am. 108, pp. 2796-2815 (2000).

[12] K.L. Williams, "An effective density fluid model for acoustic propagation in sediments derived from Biot theory," J. Acoust. Soc. Am. 110, pp. 2276-2281 (2001).

[13] E.L. Hamilton, "Geoacoustic modeling of the sea floor," $J$. 
Acoust. Soc. Am. 68, pp. 1313-1340 (1980).

[14] E.L. Hamilton and R.T. Bachman, "Sound velocity and related properties of marine sediments," J. Acoust. Soc. Am. 72, pp. 1891-1904 (1982).

[15] E.L. Hamilton, "Prediction of in-situ acoustic and elastic properties of marine sediments," Geophysics 36, pp. 266-284 (1971).

[16] P.S. Wilson, R.A. Roy, and W.M. Carey, "An improved water-filled impedance tube," J. Acoust. Soc. Am. 113, pp. 3245--3252 (2003).

[17] L.E. Kinsler, A.R. Frey, A.B. Coppens, and J.V. Sanders, Fundamentals of acoustics, 4rd ed. New York: Wiley, 2000. 